Original Research Paper

\title{
Structural Optimization for Materially Informed Design to Robotic Production Processes
}

\author{
H. Bier and S. Mostafavi \\ Hyperbody-Robotic Building, TUD, Delft, The Netherlands
}

\section{Article history}

Received: 27-07-2015

Revised: 20-08-2015

Accepted: 03-09-2015

Corresponding Author:

H. Bier

Hyperbody-Robotic Building,

TUD, Delft, The Netherlands

Email: h.h.bier@tudelft.nl

\begin{abstract}
Hyperbody's materially informed Design-to-Robotic-Production (D2RP) processes for additive and subtractive manufacturing aim to achieve performative porosity in architecture at various scales. An extended series of D2RP experiments aiming to produce prototypes at 1:1 scale wherein design materiality has been approached from both digital and physical perspectives were recently implemented. At digital materiality level, a customized computational design framework for compression only structures has been developed, which was directly linked to the robotic production setup. This has enabled the systematic study of physical materiality, which cannot be fully simulated in the digital medium. The established feedback loop ensured not only the development of an understanding for material properties in relation to their simulated and real behaviours but also allowed to robotically additively deposit and/or subtractively remove material in order to create informed material architectures at 1:1 scale.
\end{abstract}

Keywords: Materially Informed Design, Robotic Production, Topological Optimization, Material Behavior

\section{Introduction}

The Design-to-Robotic-Production (D2RP) framework developed at Hyperbody TUD employs customized processes that incorporate material properties in design, control all aspects of these processes numerically and link parametric models with robotized production tools in order to achieve efficient production of customizable building components. This materially informed D2RP framework exploits the potential of robotic fabrication techniques to innovate generative design to materialization practices.

Extensive experimentation with optimized additive and subtractive D2RP of compression-only computationally derived topologies was implemented with focus on the design and fabrication of material architectures that have various levels of porosities, ranging from architectural (macro) to componential (meso) and material (micro) scales. By employing performative and generative design methods, robotic production techniques and material science experiments, the D2RP process aimed at establishing a feedback loop between computational design and robotic production at 1:1 scale.

The D2RP process required translation of the structural optimization results from a finite geometry into continuous and discontinuous robotic paths for material deposition or subtraction in order to create material architectures.

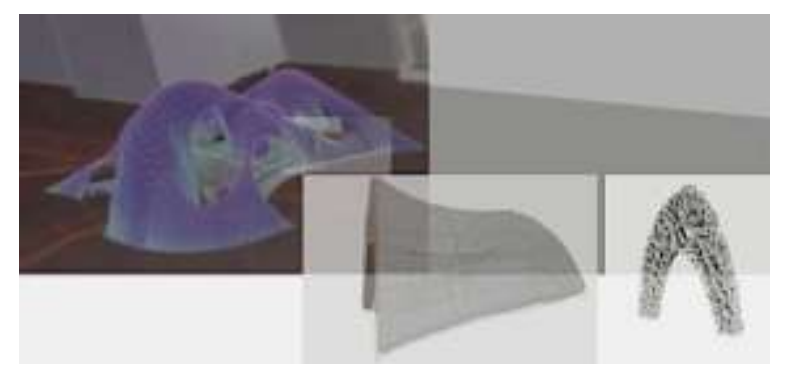

Fig. 1. Additive D2RP-Fragment of urban furniture presenting porosities at architectural, component and material scales

The integration of physical material properties into design by means of computation has been explored in both practice and academia (Kolarevic, 2005; Gramazio and Kohler, 2008). Two major types of approaches have been identified: One in which the design system relies on virtual modelling, simulation, analysis and abstraction of material physicality by means of computational methods such as Finite Element Method (FEM), Computational Fluid Dynamics (CFD), Particle Systems, etc. and the 
other one integrates constraints of material and/or fabrication methods into digital modelling platforms, i.e., parametric design models. Hyperbody's D2RP system establishes a link between the two approaches. At digital materiality level, a systematic and chained strategy for design information exchange is established by combining a customized parametric form finding approach for compression-only structures with topology optimization. At physical level, the robotic production system explores physicality that cannot be fully modelled inside the digital design platform in order to inform the design. Therefore, the D2RP system connects physical fabrication with virtual modeling and simulation.

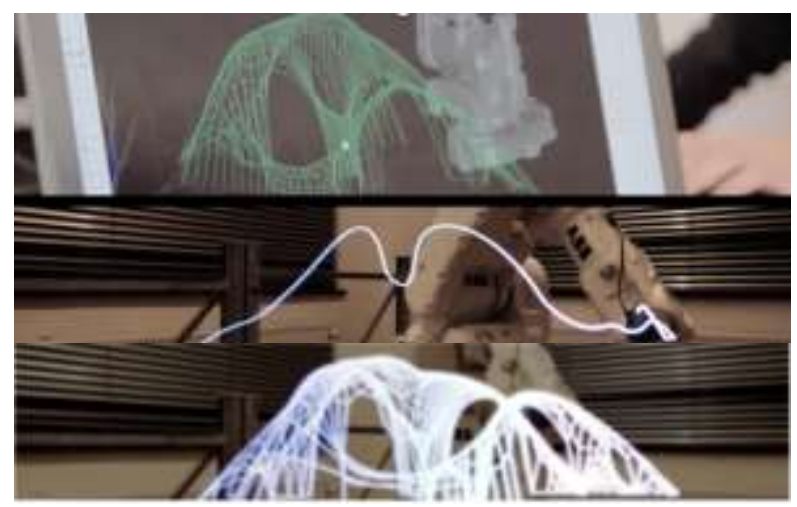

Fig. 2. Additive D2RP explored transfer from virtual to physical path control via a light experiment

This paper presents the D2RP approach by reviewing additive and subtractive D2RP experiments implemented 2014-15 at Hyperbody, TU Delft. The objective is to identify potential and limitations of this approach for the further development of multi-materials, -tools and robots to be implemented incrementally in the next phases of this research.

\section{D2RP System}

D2RP addresses customization as a process, which exploits the potential of todays interlacing virtual and physical environments, wherein robots are used in a wide range of production processes. More recently architectural research and practice started to explore their potential for architecture. For instance, Gramazio and Kohler (2014) and Menges (Krieg and Menges, 2013) employ industrial robots for developing 1:1 prototypes that explore the potential of robotic production in architecture. Similarly, Hyperbody develops 1:1 prototypes but focuses on the building and building process in a holistic way looking at the complete design to production and operation chain. RB does not separate the design to production process from operation; the opposite, it integrates operation from the early stages of design. It, furthermore, employs industrial robots with customized end-effectors in design to production processes and embeds customized robotic devices into robotic buildings.

In this context, D2RP drafts a roadmap for development of customizable 1:1 building components. This roadmap has been tested in two case studies dealing with additive and subtractive robotic techniques, respectively. Both have potentials and limitations that have been explored in order to later develop multi-tools, -materials and -robots approaches for building on demand.

\section{Additive D2RP}

Recent advances in robotics have introduced new approaches to architectural materialization. Considering materiality and architecture at multiple scales, there are a few examples that bring the two together. For instance, a scaled up printing machine is horizontally depositing layer-by-layer a cement-based building material (Khoshnevis, 2001; Dini et al., 2006; Kestelier, 2012) without taking locally changing structural and climatic constraints into account. Hyperbody's D2RP project proposes an alternative method of additive material deposition by integrating local and global material optimization routines in the process while taking the simulated and real behaviors and properties of the material into consideration.

Customizability of the production method, while taking both research and design objectives into consideration, has enabled the development of a unique D2RP system. The design methodology implied robotic motion path generation in relation to material properties and the robotically produced 1:1 prototype.

Basically, D2RP links design computation, tooling and robotics, with material research. D2RP experiments and design exercises implemented since 2014 explored possibilities of integration by establishing feedback loops between the individual components. The first case study (Fig. 1) explored architectural and material porosity at various scales and the developed D2RP system was customized and implemented according to the particular research and design objectives of the project.

Light robotic path drafting, as the first preliminary study for translating digital geometry into robotic tool paths, involved mounting a color changing light source on the robotic arm. The connection established between 6-axes-robot motion and information extracted from the virtual 3D model contributed to the development of a new approach to $3 \mathrm{D}$ printing, different than the established contour crafting technique, which implies the slicing of the geometry in horizontal layers. The new approach aimed to be more in tune with the structural characteristics of the physical prototype. 
The study of robotic motion defined the boundaries of the digital design-space in relation to the physical solution-space. Furthermore it informed the parametric setup with ranges of reach ability and optimized orientations thus contributing to maximizing it. In addition, by numerically controlling the on-off light pattern and light colors with Arduino Microcontrollers, the team reached the goal of further extending design possibilities in such a way that multiple materials were deposited based on the information extracted from the virtual 3D geometry. In a the first step, any given curve, in digital, was reproduced, in physical, with multicolored light curves captured by means of long exposure-time photography. This approach was tested on the designed urban furniture e.g., pavilion represented as a network of curves (Fig. 2).

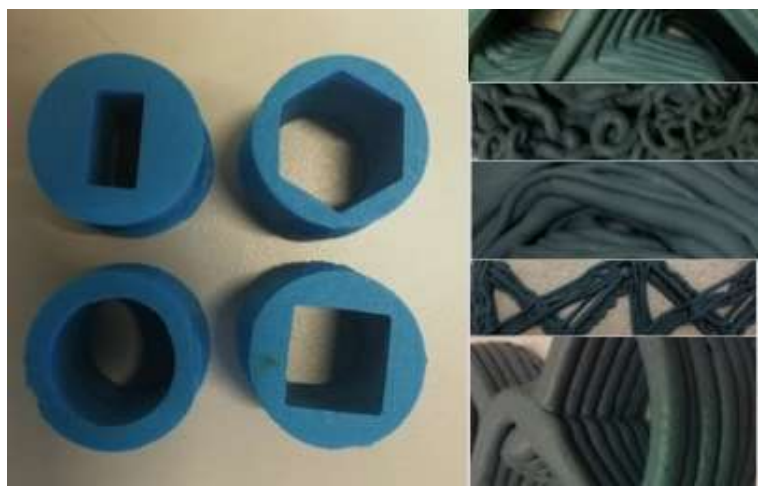

Fig. 3. Additive D2RP implies material and end-effect or (nozzle) customization in order to achieve specific material deposition

As part of the second set of preliminary studies, the robotic light pattern project focused on drawing geometric patterns that explored variation in densities and resolutions aiming to reach desired porosities. This informed the design of robotically controlled routines for material deposition. The established parametric system, derived from these experiments, involved parameters related to size of the overall shape, thickness of nozzle for material deposition, number of targets to describe robotic motion and method of approaching defined targets. In this context, two categories of material deposition were formulated: Continuous flow and on/off numerically controlled flow patterns. Both directions had specific benefits and limitations. Continuous material deposition involved a bigger abstraction of the material deposition patterns, which was compensated through the overall structural performance. The on-off numerically controlled material deposition enabled a more accurate representation of the structurally optimized material pattern but the logic used for production was rather fragmented.

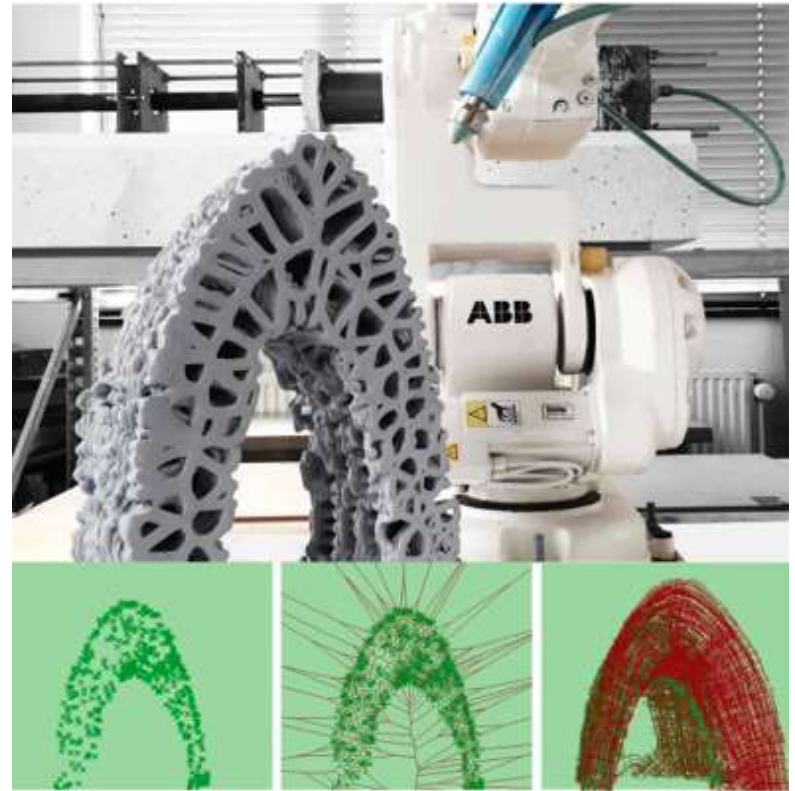

Fig. 4. Structural optimization routine for additive D2RP and 1:1 prototype of urban furniture (fragment)

The ceramic robotic 3D printing study explored possibilities of producing building components at 1:1 scale and established a D2RP method where all parameters were calibrated for the developed physical set-up. The team designed an extruder connected to an end-effect or mounted on the head of a robotic arm, where the material container was exterior to the robotic arm in order to maximize the freedom of movement. In order to achieve an optimum multidimensional material-architecture that is informed by experiments with various printing resolution and material deposition patterns, a customizable extrudernozzle system was designed and tested (Fig. 3). Considering the fact that natural materials are not fully predictable several material properties like plasticity, viscosity, flow rate and short-term material behaviors were investigated and documented at different robotmotion speeds in order to provide complete information sets for the next prototyping phase.

Form finding for compression-only structures was derived from the innate characteristics of the materials. After eliminating tension forces, the derived topology was subjected to operations aiming to achieve porosity at architectural (macro) scale to fulfill functional and formal requirements. Furthermore, in order to achieve the material (micro) porosity level, a finite element method for material distribution optimization was implemented on fragments of the designed structures.

\section{Subtractive D2RP}

The second case study employed subtractive tools and techniques as for in-stance, volumetric wire cutting 
and milling. Once again the design tasks aimed at transgressing scales (Fig. 5).

The design of student housing explored porosities at material, component and building scale (Fig. 5 and 6) related to functional, climatic and structural needs. The assumption was that material logics inform componential and building logics. Notions such as hybridity, variation and componentiality were investigated at different scales, ranging from micro levels, as material systems, to macro levels as spatial and architectural configurations.

Hybridity investigated architecture as consisting of multiple materials and systems that have various performances. Changes in performance requirements addressing, for instance, inside-outside transitions imply changes in degrees of material porosity.

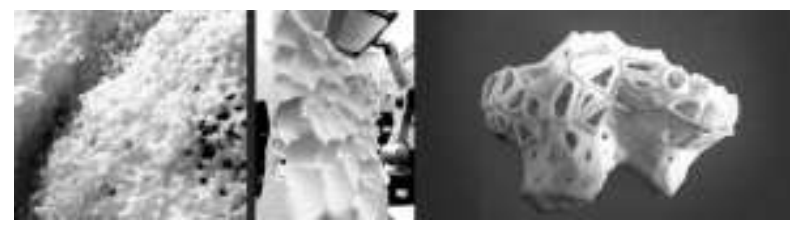

Fig. 5. Subtractive D2RP material, component, and building scale explorations

Variation implied an understanding that architecture deals with varying conditions, characteristics and aspects that are based on performative aspects (related to functional, structural and/or environmental needs) and are customized according to individual and personalized specifications.

Componentiality explored architectural systems that consist of components operating together as a distributed system, whereas building systems and components can be scaled up and down to some degree.

All three notions informed each other and were relevant in the generative design process that in the end produced an initial geometry linking material to componential and building logics. The geometry to tool path translation implied experimentation with various trajectories (linear, circular, etc.) and changing porosity patterns (Fig. 7-9).

In contrast to conventional $\mathrm{NC}$ milling, robotic subtractive techniques such as volumetric wire cutting and milling allow 3D transformation of materials as they can be approached from all sides and the tools can be freely moved in 3D space. The resulting 3D cavernous structures (Fig. 11) have optimized structural and climate control behaviors and are robotically produced from EPS, which is light, easy to wire cut or mill, is recyclable, cheap and can be easily coated for indoor and outdoor use.
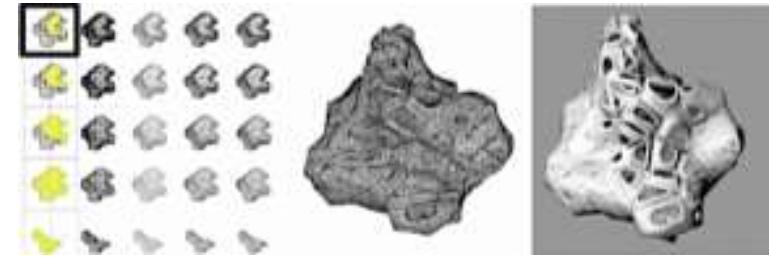

Fig. 6. Subtractive D2RP-Morphology and porosity aspects explored at component and building scale

These experiments have shown that additive and subtractive D2RP employs customizable processes that allow interaction between natural (humans) and artificial (non-human or cyber-physical) environments. In this context, architectural production results from human and non-human interaction while using multiple tools and techniques, materials and robots. The mid- and long-term goal of research in $\mathrm{D} 2 \mathrm{RP}$ is to produce on demand, improve material and energy efficiency (reduce waste) and produce customized parts and materials by following tension and compression force distribution and climate control requirements (Fig. 8 and 9).

\section{D2RP Structural Optimization}

In order to develop a coherent D2RP system specific to each project, the first step was to implement methods for form finding of compression-only structures, derived from the innate characteristics of the materials. In addition to eliminating tension forces in the derived topology, this part of the design was implemented as a parametric strategy to define the porosity at architectural (macro) scale to fulfil functional and formal requirements. Furthermore, in order to achieve the material (micro) porosity level, a finite element method for material distribution optimization was implemented on fragments of the designed structures (Fig. 4 and 10). The optimization also considered local and global load and support conditions.

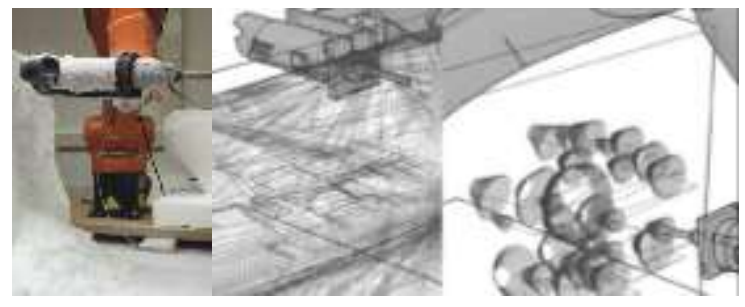

Fig. 7. Volumetric wire cutting and milling (with linear and circular paths) for subtractive D2RP

In order to develop a generic and repeatable method for other parts of the compression only structure the finite geometric representations e.g., point cloud and mesh had to be parametrically changed into vector 
based or NURBs geometry. This was achieved by applying a segmental system in the very initial topology, retrievable at different stages of form finding and parametric geometric transformation. By applying this computational design approach several configurations were generated, each distributing the compression-only material where needed and as needed, while taking the structural performance at both macro and micro scales into consideration.

The challenge of the next step in the additive D2RP case study was to materialize these differentiated densities by creating unified topologies that express structural loads consistent to the design approach and robotic fabrication potentialities and constraints, paths and targets. At this stage various algorithmic form finding and optimization techniques, mostly in Rhino-Grasshopper and Python scriptinglanguage were applied. This allowed the systematic exploration and evaluation of design alternatives in the design-solution space, eventually providing the required information for production with the $\mathrm{ABB}$ Robotstudio. At the same time, the initial material experiments informed the design.

Material optimization for robotic production informs the relevant ranges of robotic motion through parametric design control. This creates new opportunities for architectural fabrication and improves productivity and performance. For production purposes, the topology of the pavilion was sub-divided into unique components. As the research progressed it became apparent that due to the significant variety of custom building components featured in the design the robot manufacturer's software functionality needed further customization. For this purpose a link between the design and simulation environment (Rhinoceros platform and its add-ons) and the rapid code interpreter of Robotstudio has been implemented. This direct link between the design model and robot controller enabled the implementation of a greater range of unique, continuous tool paths (Fig. 3).

The additive D2RP experiment, aimed to prove that by controlling geometry and material deposition, compression-only structures could become lighter, which represents a significant improvement in their material- and energy efficiency implying cost reduction and increased thermal insulation. A way of achieving material deposition optimization is by controlling the parameters of the production setup. This is briefly described as follows: The D2RP extruder system manages a plunger-based mechanical extrusion of a diluted paste of ceramic-clay, water and color-pigment. Although numeric control of clay extrusion was experimented with and valuable results for dynamic extrusion were recorded while depositing material in a discontinuous porous pattern, this project tested solely continuous clay extrusion for the 1:1 prototype. A customized D2RP routine was developed in order to extract and optimize continuous motion path based on the designed material architecture.

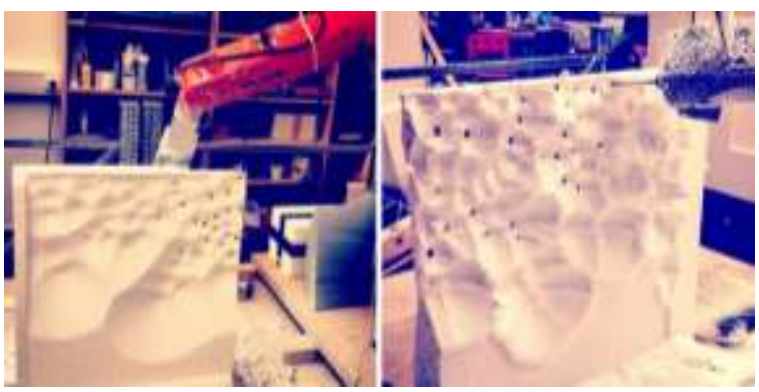

Fig. 8. Robotic 3D milling of components exhibiting geometries and porosity patterns that are informed by structural and climate control requirements

Throughout the process extrusion speed was adjusted empirically according to observed structural, functional and aesthetic considerations. Extrusion parameters were controlled through line-size and nozzle customization. These proved important factors to consider during initial experiments as well as during the fabrication routines. Nozzles of various profile-size and shape were experimented with. For the fabricated prototype, a nozzle featuring a square, 1 $\mathrm{cm} 2$ aperture was selected and used. The additive D2RP experiment proved to achieve both improved $3 \mathrm{D}$ printing speed and reliability.

The subtractive D2RP experiment employed topological optimization routines similar to the additive experiment. However, the volumetric wire cutting and milling proved to be easier to control as the material was more predictable and less dependent on environmental conditions.

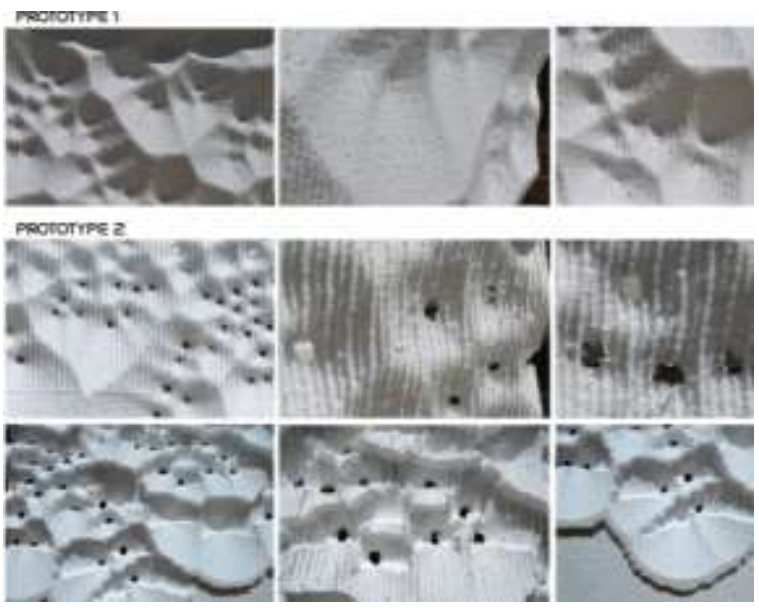

Fig. 9. Subtractive D2RP prototypes exhibiting patterns and degrees of porosity associated to spatial, structural, and climate control requirements 
The structural optimization routines enabled mapping stress lines and point clouds (Fig. 10) on the macro geometry, which allowed generation of structurally informed porous patterns. This implied that porosity at macro, meso and micro scale were implemented by mapping light, ventilation and structural needs onto the geometry.

\section{D2RP Prototypes}

At architectural level, both D2RP case studies aimed to relate architecture to the surrounding environment through pores of varying size according to functional, formal and structural requirements. The fragments chosen for fabrication exhibited porosities required for letting water sicker through so that the surface to seat on stays dry, as in the case of the urban bench, or ranges of porosities for natural lighting and ventilation, as in the case of student housing.

At material level, structural optimization was achieved in both through topological optimization.

With respect to the process, the additive D2RP employed optimization in motion path generation. Common 3D printing techniques employ nondifferentiated routines for slicing and ordering material layers into motion paths. In contrast, the D2RP process embedded fabrication potentialities and material constraints into the design. Continuous material deposition was achieved through controlled extrusion guided by robotic motion in relation to structural performance. It is notable that, although the computational 3D model comes close to the actual prototype, the two entities remain different mainly due to emergent material properties. Differences between virtual and material exemplify emergent qualities inherent to the material behavior of ceramic-clay. These qualities are as much due to the 3D layering technique as to how material extrusion varies along the path (Fig. 3 and 4).

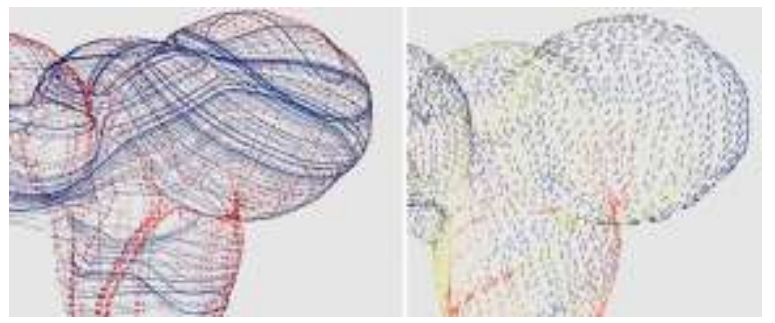

Fig. 10. Stress lines and point cloud mapped on the macro geometry establish porosity patterns at micro level

The production of subtractive D2RP prototypes has been less challenging as material removal had a rather predictable behavior. In both cases robotic prototyping established a link to building at 1:1 scale as it involved design, realization and testing at building scale. By exposing ranges for feasibility in design, fabrication and exploitation, a rapid-prototyping approach is important for avoiding failure and foreseeing D2P opportunities and threats. Furthermore, the actual integration between digital and material processes enables D2RP to bridge small and large scale.

\section{Conclusion}

Advancements in robotic building (Bier, 2014) as presented in this paper indicate that future building systems are customizable to fit demand driven production and D2RP processes enable material- and energy-efficient building. Such efficiency relies on materially, structurally and environmentally informed porosity, which at macro (building), meso (skin) and micro (material) scales, requires optimization of spatial configuration and material distribution with the aim to not only control mass-void ratios but also achieve an integral design, from overall building configuration to the achitectured material itself.

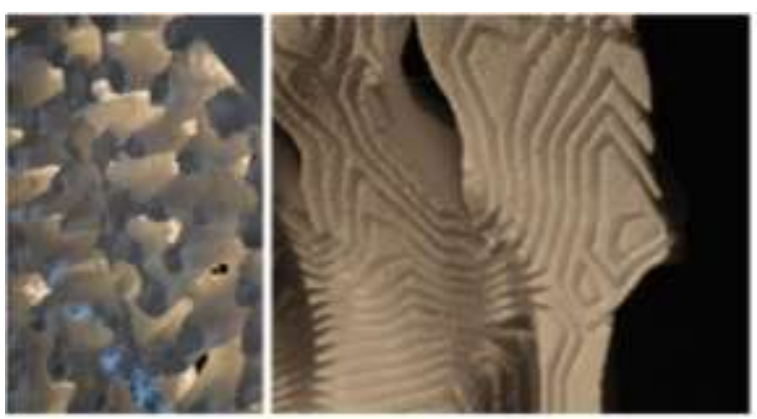

Fig. 11. Subtractive D2RP prototypes exhibiting cavernous patterns implemented by means of volumetric cutting

In both experiments, the goal to scale up robotic production was achieved by integrating the informed, chained design with the robotic production, which requires a ready to be used working-operating module. The main consideration was that in architecture and building construction, the factory of the future employs building materials and components that can be robotically processed and assembled. This requires development of multi-materials, -tools and -robots processes, to be implemented incrementally in the next phases of this research.

D2RP takes the inherent multifaceted nature of buildings into consideration from the early design to the latest building operation phase. The ultimate goal is to implement chained D2RP processes in which robots take specific roles while all (human and non-human) members of the setup as well as the respective design systems receive feedback at all time. 
In this context, both robotic additive and subtractive techniques are relevant and need to be integrated into D2P processes. While additive techniques may be slower, they have the advantage of enabling generation of highly customized material architectures. Subtractive techniques such as volumetric wire cutting and milling prove to be easier to control as material behavior is more predictable and less dependent on environmental conditions. A combination of both is envisioned to push D2RP to the next level, which aims at development of multi-material, -techniques and -robots approaches. These transform building construction at the level where product-data descriptions drive $\mathrm{NC}$ and robotic manufacturing operations and processes. Such processes for the manufacturing of customized building components combine materialization techniques aimed at optimizing structural and climate control requirements as well as improving material and production efficiency. In this context, structural optimization is not anymore a post-optimization routine, but a design driver.

D2RP builds up on developments of the third and fourth industrial revolutions (Anderson, 2012), which are increasingly enabling users to act as co-creators of architectural artifacts. The seamless data integration across the process chains for the fast production of custom made building components enables fast supply and distribution by making in the future the D2RP framework accessible to users on the Internet. Designs are then customized by users and $\mathrm{NC}$ and/or robotically produced and assembled.

\section{Acknowledgement}

This paper has profited from the contribution of Hyperbody's Robotic Building team and students.

\section{Funding Information}

This paper has been supported by TUD, 3TU Bouw, Delft Robotics Institute, 100\% Research and ABB.

\section{Author's Contributions}

First and second authors have contributed to this paper in their roles as project leader and team member, respectively, of the Robotic Building project.

\section{Ethics}

There are no known ethical issues that may arise after the publication of this manuscript.

\section{References}

Anderson, C., 2014. Makers of the New Industrial Revolution. 1st Edn., Crown Business, New York, ISBN-10: 0307720969, pp: 272.

Bier, H., 2014. Robotic building(s). Next Generat. Build., 1: 83-92. DOI: 10.7564/14-NGBJ8

Dini, E., R. Nannini and M. Chiarugi, 2006. Methods and device for building automatically conglomerate structure. WOPatent WO2006100556.

Gramazio, F. and M. Kohler, 2008. Digital Materiality in Architecture. Springer, Baden, ISBN-10: 303778122X, pp: 111.

Gramazio, F., M. Kohler and J. Willmann, 2014. Authoring robotic processes. Architectural Design, 84: 14-21. DOI: 10.1002/ad.1751

Kestelier, X.D., 2012. Design Potential for Large-Scale Additive Fabrication, Free Form Construction. Fabricate-Making Digital Architecture, Glynn, R. and B. Sheil (Eds.), Riverside Architectural Press, Cambridge, ISBN-10: 1926724186, pp: 244-249.

Khoshnevis, B., 2001. Experimental investigation of contour crafting using ceramic materials. Rapid Prototyp., 7: 32-411. DOI: $10.1108 / 13552540110365144$

Kolarevic, B., 2005. Architecture in the Digital Age: Design and Manufacturing. 2nd Edn., Taylor and Francis, New York, ISBN-10: 041538141X, pp: 320.

Krieg, O. and A. Menges, 2013. Prototyping robotic production: Development of elastically bent wood plate morphologies with curved finger joint seams. Proceedings of the Design Modelling Symposium Berlin, (MSB' 13), Verlag der Universität der Künste Berlin, pp: 479-490. 